\title{
Borboletas frugívoras da Mata Atlântica no Parque Estadual da Serra do Tabuleiro, Santa Catarina, Brasil
}

\author{
Gabriela Corso* \\ Malva I. M. Hernández \\ Universidade Federal de Santa Catarina, Centro de Ciências Biológicas \\ Departamento de Ecologia e Zoologia, Laboratório de Ecologia Terrestre Animal \\ CEP 88010-970, Florianópolis - SC, Brasil \\ * Autor para correspondência \\ gabicorso@gmail.com
}

Submetido em 01/09/2011

Aceito para publicação em 27/08/2012

\section{Resumo}

As borboletas frugívoras são utilizadas em estudos ecológicos comparativos seguindo protocolos de amostragem padronizados, pois são facilmente coletadas por armadilhas com iscas de atração alimentar. Tal característica, aliada ao fato de suas populações responderem frente a variações no hábitat, as tornam boas indicadoras biológicas. Este estudo objetivou elaborar uma lista de espécies e apresentar características ecológicas e morfológicas das borboletas frugívoras encontradas em áreas de Mata Atlântica no Parque Estadual da Serra do Tabuleiro, Santa Catarina, auxiliando no conhecimento deste grupo de borboletas para a região. Foram realizadas seis campanhas ao longo de um ano (novembro de 2009 a agosto de 2010), utilizando-se 25 armadilhas de atração em cada uma, que permaneceram ativas durante dez dias e foram revisadas a cada dois dias para reposição de isca e coleta dos indivíduos. Foram capturados 331 indivíduos de 20 espécies, pertencentes a três subfamílias: Biblidinae, Charaxinae e Satyrinae. O estudo acrescentou onze novas espécies à lista já existente para a Mata Atlântica de Santa Catarina.

Palavras-chave: Ecologia; Floresta Ombrófila Densa; Lepidoptera; Lista de espécies; Nymphalidae

\section{Abstract}

Fruit-feeding butterflies in the Atlantic Forest at Serra do Tabuleiro State Park, Santa Catarina State, Brazil. Fruit-feeding butterflies are used in ecological studies following standardized sampling protocols because they are easily collected using traps baited with food. This trait, and the fact that their populations respond rapidly to changes in habitat, make them good biological indicators. The goal of this study is to present a list of fruit-feeding butterfly species, including morphological and ecological characteristics of this group, for the Atlantic Forest of Serra do Tabuleiro State Park (Santa Catarina), to improve the knowledge about the butterflies from this region. Six field expeditions were carried out between November 2009 and August 2010. Twenty-five bait traps were used, which remained active for ten days and were checked every $48 \mathrm{~h}$ to replace the bait and collect individuals. Twenty species and 331 individuals were captured, which belonged to three subfamilies: Biblidinae, Charaxinae and Satyrinae. The study added eleven new species to the already existing list of the Atlantic Forest butterflies of Santa Catarina.

Key words: Ecology; Lepidoptera; Nymphalidae; Rain Forest; Species list 


\section{Introdução}

As borboletas correspondem a $13 \%$ de uma riqueza de 146.000 espécies de lepidópteros no mundo, sendo conhecidas no Brasil 3.288 espécies de borboletas classificadas em seis famílias: Hesperiidae, Lycaenidae, Nymphalidae, Papilionidae, Pieridae e Riodinidae (BROWN JR.; FREITAS, 1999). A família Nymphalidae é a que contém o maior número de espécies, com registros de cerca de 2.850 para a região Neotropical, das quais 788 espécies são encontradas no Brasil (BROWN JR.; FREITAS, 1999; LAMAS, 2008), pertencentes a 12 subfamílias, com espécies bastante diversas quanto à forma das asas, colorido e tipo de voo (WAHLBERG et al., 2009). Quando na forma larval, alimentam-se principalmente da parte foliar das plantas. Muitas espécies têm preferência por determinada família ou espécie de planta-hospedeira, onde a fêmea adulta coloca os ovos e as lagartas alimentam-se até o momento de entrar na fase de pupa, apresentando, assim, estreita relação com a vegetação de determinada área (SINGER, 1984; DEVRIES, 1987). Quanto à alimentação durante a fase adulta, os ninfalídeos podem ser divididos em duas guildas: as nectarívoras, que são as borboletas que se alimentam do néctar encontrado nas flores, e as frugívoras, que se alimentam de frutos fermentados, exsudados de árvores, carcaças e excrementos de mamíferos, compondo entre $40-55 \%$ das espécies da família (DEVRIES, 1987; DEVRIES et al., 1999; DEVRIES; WALLA, 2001). As borboletas frugívoras são amplamente utilizadas em estudos ecológicos comparativos seguindo protocolos de amostragem, já que são facilmente coletadas por meio de armadilhas com iscas, sendo fortemente atraídas por esse recurso (DEVRIES et al., 1997).

O conhecimento da fauna de borboletas frugívoras na região sul do Brasil teve seu início através da contribuição fundamental de antigos naturalistas, como Fritz Müller, que chegou ao Brasil em 1852 e descreveu inúmeras espécies de invertebrados, dentre as quais borboletas (BARRACO; ZILLIG, 2009); e Fritz Plaumann, o qual deixou uma coleção com mais de 17 mil espécies de insetos, depositados no Museu Entomológico Fritz Plaumann, no município de Seara (MUSEU ENTOMOLÓGICO FRITZ PLAUMANN,
2011). Atualmente, o conhecimento da fauna de borboletas da região Sul do Brasil está muito aquém do esperado, principalmente em Santa Catarina. O estado do Rio Grande do Sul mostra uma riqueza de 292 espécies na Mata Atlântica (Floresta Ombrófila Densa), pertencentes a cinco famílias, sendo a maioria das espécies da família Nymphalidae (ISERHARD; ROMANOWSKI, 2004) e cerca de 310 espécies em Floresta Ombrófila Mista e Campos de Cima da Serra (ISERHARD et al., 2010; RITTER et al., 2011). As borboletas frugívoras registradas para a Mata Atlântica do Rio Grande do Sul somam 74 espécies, sendo registradas 60 na Floresta Ombrófila Densa (SANTOS et al., 2011) e 54 espécies na Floresta Ombrófila Mista (PEDROTTI et al., 2011; SANTOS et al., 2011), com a subfamília Satyrinae a de maior representatividade em ambas formações vegetais. O conhecimento da fauna de borboletas no Paraná é o maior para a região Sul e registra aproximadamente 1.200 espécies, sendo 486 espécies registradas para a região de Curitiba (MIELKE, 1994; MIELKE; CASAGRANDE, 2004) e 689 espécies para a região de Guarapuava (DOLIBAINA et al., 2011). Já no Estado de Santa Catarina, embora os antigos naturalistas realizassem diversas coletas, não há publicações de listas de espécies, restando somente alguns registros nas coleções (SANTOS et al., 2008). Brown Jr. e Freitas (2000) mencionam 796 espécies para a região de Joinville, provenientes de dados registrados por H. Miers, O. Mielke, K. Brown Jr. e outros. Para a Ilha de Santa Catarina, há o registro de 236 espécies, sendo 74 da família Nymphalidae (CARNEIRO et al., 2008) e, posteriormente, o trabalho de Gabriela Corso, da Universidade Federal de Santa Catarina, que registrou mais oito espécies desta família que não haviam sido coletadas por Carneiro et al. (2008).

A carência de conhecimento sobre as borboletas frugívoras nas áreas de Mata Atlântica em Santa Catarina e a utilização deste conhecimento para trabalhos de monitoramento, leva ao objetivo deste trabalho: apresentar uma lista com as espécies de borboletas frugívoras, bem como as suas características ecológicas e morfológicas. 


\section{Material e Métodos}

O Parque Estadual da Serra do Tabuleiro (PEST) está localizado em Santa Catarina (entre 27०41'09"S e $28^{\circ} 12^{\prime} 42^{\prime \prime S}$ e $48^{\circ} 49^{\prime} 20^{\prime \prime} \mathrm{W}$ e $48^{\circ} 25^{\prime} 08^{\prime \prime} \mathrm{W}$ ), ocupando uma área de cerca de 90.000ha (aproximadamente $1 \%$ do território do estado), sendo a maior Unidade de Conservação de Proteção Integral neste estado. Foi criado no ano de 1975, através do Decreto ${ }^{\circ}$ 1.260/75, e abrange áreas de nove municípios (SALIÉS, 2000), compreendendo regiões de serra e de planície costeira e reunindo cinco das seis fitofisionomias presentes no estado. A vegetação da área de estudo é formada originalmente por Floresta Ombrófila Densa Sub-Montana, situada entre 30 e 400m de altitude, caracterizada pela presença de canela-preta (Ocotea catharinensis), peroba (Aspidosperma olivaceum) e pau-óleo (Copaifera trapezifolia) (REIS et al., 2000). O clima na área de estudo é influenciado por duas massas de escala regional: massa polar (fria e úmida) e massa tropical atlântica (quente e úmida), caracterizando o clima mesotérmico úmido, com verão quente, nas áreas abaixo de $800 \mathrm{~m}$ e verão brando nas montanhas acima de $800 \mathrm{~m}$ (PIMENTA, 2000). A pluviosidade anual gira em torno dos $1.700 \mathrm{~mm}$, sendo os meses com maior pluviosidade janeiro, fevereiro e março, e os meses do outono e inverno os com menores índices de pluviosidade (PIMENTA, 2000).

A amostragem das borboletas frugívoras foi realizada em seis campanhas, das quais quatro abrangeram as diferentes estações do ano (novembro de 2009, fevereiro, maio e agosto de 2010) e duas foram realizadas em março e abril de 2010, dentro do período de maior abundância esperada para as borboletas (setembro a março), segundo Ribeiro et al. (2010). Foram utilizadas armadilhas cilíndricas tipo VanSomeren-Rydon modificadas (RYDON, 1964; UEHARA-PRADO et al., 2005) com isca atrativa; a isca utilizada foi banana fermentada com caldo de cana durante $48 \mathrm{~h}$, colocada em copos plásticos, presos na base da armadilha (Figura 1). Em cada campanha foram utilizadas 25 armadilhas, instaladas em grupos de cinco, distanciadas aproximadamente $20 \mathrm{~m}$ entre si e a uma altura de cerca de $1 \mathrm{~m}$ do chão. Cada grupo de armadilhas foi colocado em uma área de Mata Atlântica em diferentes estados de conservação (totalizando cinco áreas), distantes em torno de 500m umas das outras. As armadilhas ficaram ativas durante dez dias no campo em cada campanha, sendo checadas a cada $48 \mathrm{~h}$ para reposição da isca e retirada dos indivíduos capturados (HUGHES et al., 1998). Em cada campanha, o esforço amostral total foi de 250 armadilhas-dia, com um total geral de 1.500 armadilhas-dia ao final do trabalho.

FIGURA 1: Armadilha do tipo VanSomeren-Rydon modificada, para captura de borboletas frugívoras.

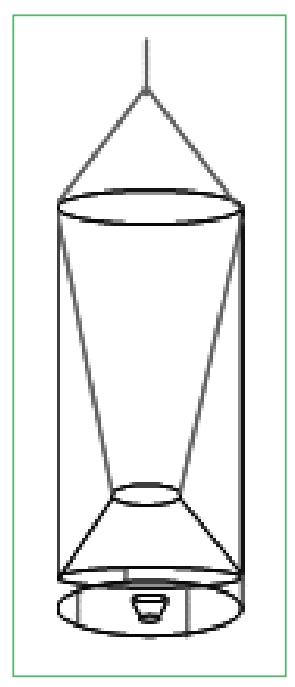

As áreas de amostragem localizavam-se em três trilhas diferentes e foram escolhidas de acordo com seu estado de conservação. As áreas apresentam um conhecido histórico de desmatamento, devido a um surto de febre amarela ocorrido no estado na década de 1950 , por isso a facilidade em definir áreas de mata secundária, que já sofreram desmatamento, e áreas de mata primária. Na trilha próxima à Pousada da Mata, conhecida como "Estrada Velha" - área de mata secundária utilizada por funcionários e hóspedes - foram alocadas cinco armadilhas. Também próxima à Pousada, a trilha conhecida como "grotão" foi escolhida por manter ainda uma vegetação de mata conservada, ou "mata primária", apresentando árvores maiores, menor quantidade de arbustos e maior quantidade de epífitas, sendo alocadas nessa área mais cinco armadilhas. Na trilha conhecida como Cascata do Tatu, foram escolhidas mais três áreas. No início da trilha escolheu-se uma área de capoeirinha, bastante degradada, com muita luminosidade e muitos arbustos, onde foram colocadas cinco armadilhas. $\mathrm{Na}$ 
continuação da trilha, em uma área de mata secundária, foram alocadas mais cinco armadilhas. E a ultima área escolhida, nessa mesma trilha, foi uma de mata primária, com árvores bastante frondosas, muitas bromélias e presença de Canela-preta, onde foram alocadas as últimas cinco armadilhas.

As borboletas capturadas foram armazenadas em envelopes entomológicos e mortas através de armazenamento em recipiente fechado contendo algodão embebido em clorofórmio. Algumas, cuja identificação era possível de ser realizada em campo, foram marcadas com caneta permanente na base da asa, de acordo com a área de captura, e posteriormente soltas. Indivíduos marcados, quando recapturados não foram contabilizados. Os indivíduos coletados foram levados para o Laboratório de Ecologia Terrestre Animal (LECOTA/UFSC), onde foram montados e secos em estufa a $40^{\circ} \mathrm{C}$ por dois dias e posteriormente depositados na Coleção Entomológica do Centro de Ciências Biológicas da Universidade Federal de Santa Catarina. A identificação foi realizada através da literatura disponível (DEVRIES, 1987; BROWN JR., 1992; CANALS, 2003; LAMAS, 2004; UEHARA-PRADO et al., 2004) e com a ajuda do Dr. André Victor Lucci Freitas, da Universidade Estadual de Campinas, através de fotos digitais.

\section{Resultados e Discussão}

Foram coletadas 20 espécies de borboletas frugívoras e um total de 331 indivíduos, pertencentes a três subfamílias de Nymphalidae, nas áreas de Mata Atlântica do Parque Estadual da Serra do Tabuleiro (Tabela 1). As descrições das subfamílias e das espécies registradas estão apresentadas a seguir, de acordo com a literatura e com os conhecimentos obtidos durante o desenvolvimento do trabalho; os dados sobre as plantashospedeiras foram retirados da bibliografia (Tabela 2).

\section{Subfamília Biblidinae}

Dividem-se em seis tribos com características morfológicas e comportamentais bastante heterogêneas, e nem todas as espécies são estritamente frugívoras. Muitas espécies têm coloração disruptiva e outras apresentam coloração críptica (CANALS, 2003).
Catonephele acontius (Linnaeus, 1771). Pertence à tribo Biblidini e possui dimorfismo sexual bem acentuado, sendo o macho preto com padrões laranja forte e as fêmeas pretas com bandas transversais amarelas (CANALS, 2003). No PEST foram encontradas nas quatro estações do ano, com maior abundância no final do verão e distribuídas principalmente em áreas de mata pouco conservada.

\section{Subfamília Charaxinae}

Possuem voo ríspido e normalmente utilizam os estratos médios e altos da vegetação para a locomoção. Muitas possuem coloração críptica na face ventral, imitando folhas secas e troncos (CANALS, 2003). São bastante agressivas, mesmo perante predadores (BROWN JR., 1992).

Archaeoprepona demophon (Linnaeus, 1758). Espécie bem distribuída do nível do mar até $1.600 \mathrm{~m}$ de altitude em associação com florestas primárias e em estágio secundário de sucessão (DEVRIES, 1987). No PEST foi coletado um indivíduo no início do outono, em área de mata degradada.

Archaeoprepona meander (Cramer, 1775). Espécie escassa em florestas tropicais (BROWN JR., 1992). No PEST foi encontrado um indivíduo no inverno, em área de mata degradada.

Fountainea ryphea (Cramer, 1775). Possuem dimorfismo sexual (macho sem cauda, fềmea com cauda marginal), e um voo vigoroso e alto (CANALS, 2003). No PEST foi coletado um indivíduo no início do outono, em área de mata degradada.

Memphis hirta (Weymer, 1907). No PEST foi coletado um indivíduo durante o inverno, em área de mata degradada. 
TABELA 1: Borboletas Nymphalidae frugívoras registradas de novembro de 2009 a agosto de 2010 em cinco áreas de Mata Atlântica no Parque Estadual da Serra do Tabuleiro (PEST), Santa Catarina, Brasil. MCT: Mata Conservada "Cascata do Tatu"; MCG: Mata Conservada "Grotão"; MSV: Mata Secundária "Estrada Velha"; MST: Mata Secundária "Cascata do Tatu"; MDT: Mata Degradada "Cascata do Tatu".

\begin{tabular}{|c|c|c|c|c|c|c|c|}
\hline \multirow{2}{*}{ Espécie } & \multirow{2}{*}{ Registro } & \multicolumn{5}{|c|}{ Áreas de coleta } & \multirow{2}{*}{ TOTAL } \\
\hline & & MCT & MCG & MSV & MST & MDT & \\
\hline $\begin{array}{l}\text { Archaeoprepona demophon } \\
\text { (Linnaeus, 1758) }\end{array}$ & & 0 & 0 & 0 & 0 & 1 & 1 \\
\hline $\begin{array}{l}\text { Archaeoprepona meander (Cramer, } \\
1775)^{1}\end{array}$ & & 0 & 0 & 0 & 1 & 0 & 1 \\
\hline Caligo beltrao (Illiger, 1801) ${ }^{1}$ & & 1 & 1 & 0 & 0 & 0 & 2 \\
\hline Caligo brasiliensis (C. Felder, 1862) & & 1 & 1 & 0 & 0 & 0 & 2 \\
\hline Caligo martia (Godart, [1824]) $)^{1}$ & & 0 & 0 & 0 & 0 & 1 & 1 \\
\hline $\begin{array}{l}\text { Catonephele acontius (Linnaeus, } \\
1771)^{2}\end{array}$ & & 1 & 1 & 1 & 7 & 2 & 12 \\
\hline $\begin{array}{l}\text { Dasyophthalma creusa (Hübner, } \\
\text { [1821]) }\end{array}$ & & 1 & 5 & 2 & 0 & 1 & 9 \\
\hline $\begin{array}{l}\text { Dasyophthalma rusina (Godart, } \\
\text { [1824]) }\end{array}$ & & 0 & 1 & 0 & 0 & 0 & 1 \\
\hline $\begin{array}{l}\text { Euptychoides castrensis (Schaus, } \\
1902)^{1}\end{array}$ & & 0 & 0 & 0 & 0 & 1 & 1 \\
\hline $\begin{array}{l}\text { Forsterinaria quantius (Godart, } \\
[1824])^{2}\end{array}$ & & 0 & 0 & 0 & 1 & 1 & 2 \\
\hline Fountainea ryphea (Cramer, 1775)² & & 0 & 0 & 0 & 0 & 1 & 1 \\
\hline Godartiana muscosa (Butler, 1870) ${ }^{2}$ & & 0 & 1 & 2 & 3 & 0 & 6 \\
\hline Memphis hirta (Weymer, 1907) ${ }^{1}$ & & 0 & 0 & 0 & 1 & 0 & 1 \\
\hline Morpho epistrophus (Fabricius, 1796) & & 68 & 43 & 23 & 54 & 58 & 246 \\
\hline
\end{tabular}




\begin{tabular}{|c|c|c|c|c|c|c|}
\hline Morpho helenor (Cramer, 1776) & 0 & 0 & 1 & 0 & 1 & 2 \\
\hline Opoptera sulcius (Staudinger, 1887) & 5 & 8 & 2 & 16 & 8 & 39 \\
\hline Opsiphanes quiteria (Stoll, 1780) & 0 & 0 & 0 & 0 & 1 & 1 \\
\hline $\begin{array}{l}\text { Pareuptychia ocirrhoe (Fabricius, } \\
1776)^{2}\end{array}$ & 0 & 0 & 0 & 0 & 1 & 1 \\
\hline $\begin{array}{l}\text { Paryphthimoides grimon (Godart, } \\
[1824])^{1}\end{array}$ & 0 & 1 & 0 & 0 & 0 & 1 \\
\hline Taygetis acuta Weymer, $1910^{2}$ & 0 & 0 & 1 & 0 & 0 & 1 \\
\hline
\end{tabular}

${ }^{1}$ Imagens cedidas por Jessie Pereira dos Santos; ${ }^{2}$ Exemplares da coleção do Museu de Zoologia da Universidade de Campinas "Professor Adão José Cardoso"

\section{Subfamília Satyrinae, Tribo Brassolini}

São em sua maioria de hábitos crepusculares e vespertinos, com um voo bastante vigoroso. Possuem ocelos na face ventral das asas.

Caligo beltrao (Illiger, 1801). As borboletas deste gênero são conhecidas como os gigantes dos Neotrópicos, sendo que nenhuma delas possui envergadura menor que $10 \mathrm{~cm}$. Voam nos horários crepusculares, tanto matutinos quanto vespertinos. A espécie C. beltrao é facilmente criada em cativeiro (BROWN JR., 1992), as lagartas demoram cerca de 70 dias até empuparem e o adulto chega a viver cerca de cinco meses em cativeiro (obs. pessoal). No PEST foram encontrados somente dois indivíduos no mês de fevereiro, em áreas em bom estado de conservação.

Caligo brasiliensis (C. Felder, 1862). São conhecidas como borboletas coruja. Sua fase de ovo dura cerca de 20 dias, com as lagartas empupando cerca de dois meses após a eclosão dos ovos (obs. pessoal). No PEST foi encontrado um indivíduo em área de mata conservada, no mês de agosto. Na Ilha de Santa Catarina é facilmente observada em áreas de mata nos meses de verão.

Caligo martia (Godart, [1824]). As borboletas desta espécie voam durante períodos de sol intenso e possuem uma geração por ano, emergindo em dezembro (CASAGRANDE; MIELKE, 2000a). No PEST foi coletado um indivíduo em março, em área de mata degradada.

Dasyophthalma creusa (Hübner, [1821]). Fêmeas e machos possuem pequenas diferenças morfológicas (PENZ, 2009). Possuem somente uma geração por ano, no verão (CASAGRANDE; MIELKE, 2003) e têm o hábito de voarem nos horários de sol intenso, rapidamente em áreas abertas e mais lentamente dentro da mata (CASAGRANDE; MIELKE, 2003). No PEST foi coletada no final do verão e início do outono, principalmente em áreas de mata conservada.

Dasyophthalma rusina (Godart, [1824]). Fêmeas e machos desta espécie apresentam pequeno dimorfismo sexual (PENZ, 2009). Possuem voo lento, nos horários de sol intenso e têm somente uma geração ao ano, no verão (CASAGRANDE; MIELKE, 2000b). No PEST foi encontrado um único indivíduo no início do outono, em área de floresta conservada.

Opoptera sulcius (Staudinger, 1887). As borboletas desta espécie possuem hábitos crepusculares. No PEST foi encontrada durante o verão e o outono, principalmente em áreas de mata em estágio intermediário de conservação. 
Opsiphanes quiteria (Stoll, 1780). No PEST, foi encontrado um indivíduo no outono, em área de mata degradada.

\section{Subfamília Satyrinae, Tribo Morphini}

Muitas espécies desta subfamília possuem tamanho grande e cores brilhantes, geralmente em tons de azul; por isso já foram bastante visadas por colecionadores (BROWN JR., 1992; CANALS, 2003). Seu voo é ondulante ou planado, e pode haver dimorfismo sexual marcado entre machos e fêmeas (DEVRIES et al., 2010); por possuírem asas proporcionalmente maiores que seu corpo, possuem a capacidade de planar sem dificuldade (CANALS, 2003). Apresentam dimorfismo sexual em relação à coloração e também de comportamento. Desaparecem rapidamente quando há perturbação forte, derrubada, ou redução em tamanho da floresta (BROWN JR., 1992).

Morpho epistrophus (Fabricius, 1796). Possui voo lento e ondulante no sub-bosque, entre as árvores ou nas bordas da floresta (DEVRIES et al., 2010). No PEST, foi a espécie mais abundante, encontrada no final do verão e início do outono, em todas as áreas do Parque.

Morpho helenor (Cramer, 1776). Possuem voo em ziguezague no sub-bosque e ao longo de rios e bordas de floresta (DEVRIES et al., 2010). Na ilha de Santa Catarina, esta espécie tem uma alta abundância, sendo encontrada preferencialmente em ambiente de floresta (trabalho de Gabriela Corso, da Universidade Federal de Santa Catarina - dados não publicados). No PEST foram encontrados dois indivíduos no início do outono em ambientes de mata pouco conservada.

\section{Subfamília Satyrinae, Tribo Satyrini, Subtribo Euptychiina}

De tamanho pequeno ou médio, possuem coloração críptica, amarronzada e algumas apresentam ocelos. Tem comportamento de permanecerem escondidas, se assemelhando ao substrato. Têm voo provocado e arrítmico (BROWN JR., 1992; CANALS, 2003). São principalmente Neotropicais (PEÑA et al., 2010).
Euptychoides castrensis (Schaus, 1902). No PEST, foi encontrado um único indivíduo no início do outono na área de mata.

Forsterinaria quantius (Godart, [1824]). No PEST foram encontrados dois indivíduos no final da primavera, em áreas de menor conservação.

Godartiana muscosa (Butler, 1870). As borboletas desta espécie preferem lugares sombrios perto de trilhas e pousam a baixa altura ou no solo (CANALS, 2003). No PEST foi encontrada no final da primavera, em áreas de estágio intermediário de conservação.

Pareuptychia ocirrhoe (Fabricius, 1776). No PEST, foi encontrado um indivíduo no outono em área em menor estado de conservação.

Paryphthimoides grimon (Godart, [1824]). No PEST, foi encontrado um indivíduo no outono em área conservada.

Taygetis acuta Weymer, 1910. Indivíduos desta espécie preferem áreas sombreadas e possuem voo arrítmico, a baixa altura (CANALS, 2003). No PEST, foi encontrado um indivíduo no inverno, em área de conservação intermediária.

Este trabalho foi o primeiro estudo de borboletas frugívoras realizado no Parque Estadual da Serra do Tabuleiro, contribuindo para o conhecimento deste grupo no maior Parque Estadual de Santa Catarina. Somam-se 11 espécies novas às listas já existentes, nos trabalhos de Carneiro et al. (2008) e Gabriela Corso, da Universidade Federal de Santa Catarina (dados não publicados). Sendo assim, este artigo serve como base para o estudo das borboletas frugívoras da região, com informações sobre a composição e características das espécies presentes, podendo ser usado como um guia na identificação das espécies, e orientar trabalhos em outras regiões do Parque. 
TABELA 2: Relação das espécies de borboletas frugívoras do Parque Estadual da Serra do Tabuleiro e suas plantas hospedeiras.

\begin{tabular}{|c|c|}
\hline Espécie & Plantas Hospedeiras (Famílias) \\
\hline Archaeoprepona demophon ${ }^{l}$ & $\begin{array}{l}\text { - Annonaceae (Annona spp.) } \\
\text { - Lauraceae (Ocotea sp. e Persea americana) } \\
\text { - Meliaceae (Guarea sp.) } \\
\text { - Monimiaceae (Mollinedia brasiliensis, M. clavigera, Siparuna sp., S. guianensis) } \\
\text { - Poraceae (Brosimum gaudichaudi) } \\
\text { - Polygonaceae (Triplaris sp.) }\end{array}$ \\
\hline Archaeoprepona meander ${ }^{l}$ & - Lauraceae (Nectandra venulosa e Persea americana) \\
\hline Caligo beltrao $^{2}$ & $\begin{array}{l}\text { - Cannaceae (Canna indica) } \\
\text { - Cyperaceae } \\
\text { - Heliconiaceae (Heliconia sp. “caeté”) } \\
\text { - Marantaceae (Calathea zebrina (Sims)) } \\
\text { - Musaceae (Musa sapientum) } \\
\text { - Zingiberaceae (Hedychium coronarium ("lírio do brejo")) } \\
\text { - Talvez Arecaceae e Strelitziaceae }\end{array}$ \\
\hline Caligo brasiliensis ${ }^{l}$ & $\begin{array}{l}\text { - Heliconiaceae (Heliconia sp. (“caeté”)) } \\
\text { - Musaceae (Musa sp.) } \\
\text { - Arecaceae (Euterpe edulis) } \\
\text { - Zingiberaceae (Hedychium coronarium e Hedychium sp.) }\end{array}$ \\
\hline Caligo martia ${ }^{1,2}$ & $\begin{array}{l}\text { - Cannaceae (Canna brasiliensis) } \\
\text { - Poaceae (Echinochloa crusgalli, E. crusgalli crusgalli, Pennisetum purpureum) }\end{array}$ \\
\hline Catonephele acontius ${ }^{l}$ & $\begin{array}{l}\text { - Euphorbiaceae (Alchornea iricurana, A. triplinervia, Aparisthmium cordatum, Nectandra venulosa } \\
\text { e Lysiloma sp.) }\end{array}$ \\
\hline Dasyophthalma creusa ${ }^{l}$ & $\begin{array}{l}\text { - Poaceae ("taquara") } \\
\text { - Arecaceae (Astrocaryum aculeatum, Bactris sp.) }\end{array}$ \\
\hline Dasyophthalma rusina ${ }^{1,2}$ & $\begin{array}{l}\text { - Poaceae (Bambusa sp., "bambu") } \\
\text { - Arecaceae (Bactris tomentosa e Euterpe edulis) }\end{array}$ \\
\hline Euptychoides castrensis ${ }^{l}$ & - Cyperaceae \\
\hline Forsterinaria quantius $^{1}$ & - Poaceae ("bambus”) \\
\hline Fountainea ryphea ${ }^{l}$ & - Euphorbiaceae (Croton sp., C. compressus, C. floribunda, C. priscus, C. urucurana) \\
\hline Godartiana muscosal & $\begin{array}{l}\text { - } \text { Cyperaceae } \\
\text { - Poaceae (Setaria poiretiana) }\end{array}$ \\
\hline Memphis hirta & - Não foram encontrados registros \\
\hline Morpho epistrophus ${ }^{1}$ & $\begin{array}{l}\text { - Erythroxylaceae (Erythroxylum sp., E. pelleterianum) } \\
\text { - Leguminosae (Cassia sp., Acacia longifolia, Inga sp., I. affinis, I. bahiensis, I. edulis, I. semialata, } \\
\text { I. sessilis, I. uraguensis, Dalbergia brasiliensis, Machaerium sp., M. acutifolium) } \\
\text { - Quiinaceae (Quiina } \text { sp.) } \\
\text { - Rhamnaceae (Scutia buxifolia) } \\
\text { - Sapindaceae (Cupania vernalis, Matayba } \text { sp.) }\end{array}$ \\
\hline Morpho helenor ${ }^{4,5}$ & $\begin{array}{l}\text { - Fabaceae (gêneros Arachis, Dalbergia, Erythrina, Lonchocarpus, Machaerium, Mucuna, } \\
\text { Platymiscium, Pithecellobium, Pterocarpus, Swartzia) } \\
\text { - Mimosaceae (gênero Inga) }\end{array}$ \\
\hline Opoptera sulcius ${ }^{1}$ & - Arecaceae \\
\hline Opsiphanes quiteria $a^{1,2,3}$ & 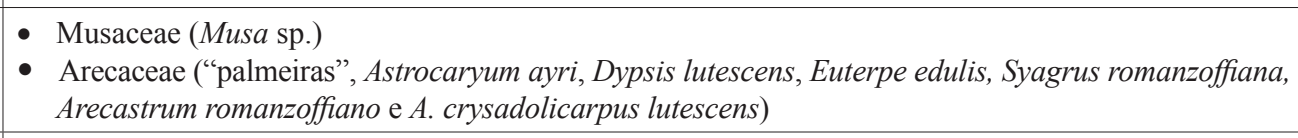 \\
\hline Pareuptychia ocirrhoe $^{l}$ & - Poaceae (Setaria sp.) \\
\hline Paryphthimoides grimon ${ }^{l}$ & - Poaceae \\
\hline Taygetis acutal & - Poaceae \\
\hline
\end{tabular}

${ }^{1}$ Beccaloni et al., 2008; ${ }^{2}$ Penz et al., 1999; ${ }^{3}$ Casagrande e Mielke, 2005; ${ }^{4}$ Ackery, 1988; ${ }^{5}$ Constantino e Corredor, 1997. 


\section{Agradecimentos}

Agradecemos ao Dr. André V. L. Freitas (UNICAMP) pela ajuda na identificação das espécies; ao biólogo Artur Nishibe Furegatti e ao Dr. André V. L. Freitas pela autorização de visita ao Museu de Zoologia da Universidade de Campinas "Professor Adão José Cardoso"; ao mestrando Jessie P. dos Santos (UNICAMP) por ter cedido algumas fotos para este trabalho e ao Dr. Cristiano A. Iserhard (UNICAMP) pela ajuda na visita à coleção do Museu; aos integrantes do Laboratório de Ecologia Terrestre Animal (LECOTAUFSC) pela ajuda no campo; ao biólogo Fernando Brüggemann e ao Plaza Caldas da Imperatriz Resort e SPA pelo apoio logístico e autorização para realização do trabalho na área; à CAPES pela bolsa de mestrado concedida a G. Corso; ao Programa de Pós-Graduação em Ecologia da Universidade Federal de Santa Catarina pelo apoio financeiro.

\section{Referências}

ACKERY, P. R. Hostplants and classification: a review of nymphalid butterflies. Biological Journal of the Linnean Society, London, v. 33, 95-203, 1988 .

BARRACO, M. A.; ZILLIG, C. Parceiro de Charles Darwin. 2009. Disponível em: $<$ http://www2.uol.com.br/sciam/reportagens/ parceiro_de_charles_darwin.html $>$.Acesso em: 20 maio 2011.

BECCALONI, G. W.; VILORIA, A. L.; HALL, S. K.; ROBINSON, G. S. Catalogue of the hostplants of the neotropical butterflies. Catálogo de las plantas huésped de las mariposas neotropicales. v. 8. Monografias Tercer Milênio, S.E.A., Zaragoza: RIBES, CYTED, The Natural History Museum \& Instituto Venezolano de Investigaciones Científicas, 2008. $536 \mathrm{p}$.

BROWN JR., K. S. Borboletas da Serra do Japi: diversidade, habitats, recursos alimentares e variação temporal. In: MORELLATO, L. P. C. (Ed.). Historia Natural da Serra do Japi. Ecologia e preservação de uma área florestal no sudeste do Brasil. Campinas, São Paulo: Editora UNICAMP/FAPESP, 1992. p. 142-186.

BROWN JR., K. S.; FREITAS, A. V. L. Lepidóptera. In: BRANDÃO, C. R. F.; CANCELLO, E. M. (Org.). Biodiversidade do Estado de São Paulo, Brasil: síntese do conhecimento ao final do século XX, 5: invertebrados terrestres. São Paulo: Fundação de Amparo à Pesquisa do Estado de São Paulo (FAPESP), 1999. p. 227-243.

BROWN JR., K. S.; FREITAS, A. V. L. Atlantic Forest butterflies: indicator of landscape conservation. Biotropica, Zurich, v. 32, p. 934-956, 2000.

CANALS, G. R. Mariposas de Misiones. Buenos Aires: L.O.L.A., 2003. 492 p

CARNEIRO E.; MIELKE, O. H. H.; CASAGRANDE, M. M. Borboletas do sul da ilha de Santa Catarina, Florianópolis, Santa
Catarina, Brasil (Lepidoptera: Hesperioidea e Papilionoidea). SHILAP Revista de Lepidopterologia, Madrid, v. 36, p. 261-271, 2008.

CASAGRANDE, M. M.; MIELKE O. H. H. Larva de quinto estádio e pupa de Caligo martia (Godart) (Lepidoptera, Nymphalidae, Brassolinae). Revista Brasileira de Zoologia, Curitiba, v. 17, p. 75-79, 2000a.

CASAGRANDE, M. M.; MIELKE, O. H. H. Larva de quinto estádio e pupa de Dasyophthalma rusina rusina (Godart) (Lepidoptera, Nymphalidae, Brassolinae). Revista Brasileira de Zoologia, Curitiba, v. 17, p. 401-404, 2000b.

CASAGRANDE, M. M.; MIELKE, O. H. H. Larvas de quarto e quinto estádios e pupa de Dasyophthalma creusa creusa (Hübner) (Lepidoptera, Nymphalidae, Brassolinae). Revista Brasileira de Zoologia, Curitiba, v. 20, p. 157-160, 2003.

CASAGRANDE, M. M.; MIELKE, O. H. H. Larva de quinto estádio e pupa de Opsiphanes quiteria meridionalis Staudinger (Lepidoptera, Nymphalidae, Brassolinae). Revista Brasileira de Entomologia, Curitiba, v. 49, p. 421-424, 2005.

CONSTANTINO, L. M.; CORREDOR, G. The biology, and morphology of the early stages of Morpho macrophthalmus and Morpho peleides telamon (Nymphalidae: Morphinae) from western Colombia. Boletín Científico Museo Historia Natural Universidad de Caldas, Manizales Caldas, v. 8, p. 201-208, 1997.

DEVRIES, P. J. The butterflies of Costa Rica and their natural history. Princeton, New Jersey: Princeton University Press, 1987. $327 \mathrm{p}$.

DEVRIES, P. J.; MURRAY, D.; LANDE, R. Species diversity in vertical, horizontal, and temporal dimensions of a fruit-feeding butterfly community in an Ecuadorian rainforest. Biological Journal of the Linnean Society, London, v. 62, p. 343-364, 1997.

DEVRIES, P. J.; WALLA, T. R.; GREENEY, H. F. Species diversity in spatial and temporal dimensions of fruit-feeding butterflies from two Ecuadorian rainforests. Biological Journal of the Linnean Society, v. 68, p. 333-353, 1999.

DEVRIES, P.J.; WALLA, T.R. Species diversity and community structure in neotropical fruit-feeding butterflies. Biological Journal of the Linnean Society, v. 74, p. 1-15, 2001.

DEVRIES, P. J.; PENZ, C. M.; HILL, R. I. Vertical distribution, flight behaviour and evolution of wing morphology in Morpho butterflies. Journal of Animal Ecology, London, v. 79, p. 10771085, 2010.

DOLIBAINA, D. R.; MIELKE, O. H. H.; CASAGRANDE, M. M. Borboletas (Papilionoidea e Hesperioidea) de Guarapuava e arredores, Paraná, Brasil: um inventário com base em 63 anos de registros. Biota Neotropica, Campinas, v. 11, p. 1-15, 2011.

HUGHES, J. B.; DAILY, G. C.; EHRLICH, P. R. Use of fruit bait traps for monitoring of butterflies (Lepidoptera: Nymphalidae). Revista de Biología Tropical, San José, v. 46, p. 697-704, 1998.

ISERHARD, C. A.; QUADROS, M. T.; ROMANOWSKI, H. P.; MENDONÇA JR., M. S. Borboletas (Lepidoptera: Papilionoidea e Hesperioidea) ocorrentes em diferentes ambientes na Floresta Ombrófila Mista e nos Campos de Cima da Serra do Rio Grande do Sul, Brasil. Biota Neotropica, Campinas, v. 10, p. 309-320, 2010.

ISERHARD, C. A.; ROMANOWSKI, H. P. Lista de espécies de borboletas (Lepidoptera, Papilionoidea e Hesperioidea) da região 
do vale do rio Maquiné, Rio Grande do Sul, Brasil. Revista Brasileira de Zoologia, Curitiba, v. 21, p. 649-662, 2004.

LAMAS, G. Checklist: Part 4A. Hesperioidea-Papilionoidea. In: HEPPNER, J. B. (Ed.). Atlas of Neotropical Lepidoptera. Vol. 5A. Gainesville: Association for Tropical Lepidoptera/Scientific Publishers, 2004. 439 p.

LAMAS, G. La sistemática sobre mariposas (Lepidoptera: Hesperioidea y Papilionoidea) en el mundo: Estado actual y perspectivas futuras. In: LLORENTE-BOUSQUETS, J.; LANTERI, A. (Ed.). Contribuciones taxonómicas en órdenes de insectos hiperdiversos. México: Las Prensas de Ciencias, UNAM, 2008. p. 57-70.

MIELKE, C. G. C. Papilionoidea e Hesperioidea (Lepidoptera) de Curitiba e seus arredores, Paraná, Brasil, com notas taxonômicas sobre Hesperiidae. Revista Brasileira de Zoologia, Curitiba, v. 11, p. 759-776, 1994.

MIELKE, O. H. H.; CASAGRANDE, M. M. Borboletas In: MIKICH, S. B.; BERNILS, R. S. (Org.). Livro vermelho da fauna ameaçada no estado do Paraná. Curitiba: Instituto Ambiental do Paraná, 2004. p. 713-739.

MUSEU ENTOMOLÓGICO FRITZ PLAUMANN. 2011. Disponível em: $<$ http://www.museufritzplaumann.ufsc.br/>.

PEDROTTI, V. S.; BARROS, M. P.; ROMANOWSKI, H. P.; ISERHARD, C. A. Borboletas frugívoras (Lepidoptera: Nymphalidae) ocorrentes em um fragmento de Floresta Ombrófila Mista no Rio Grande do Sul, Brasil. Biota Neotropica, Campinas, v. 11, p. 1-15, 2011

PEÑA C.; NYLIN, S.; FREITAS, A.V.L.; WAHLBERG, N. Biogeographical history of the butterfly subtribe Euptychiina (Lepidoptera, Nymphalidae, Satyrinae). Zoologica Scripta, Maiden, v. 39, p. 243-258, 2010.

PENZ, C. M. Phylogeny of Dasyophthalma Butterfies (Lepidoptera, Nymphalidae, Brassolini). Insecta Mundi, Gainnesville, v. 69, p. 1-12, 2009.

PENZ, C. M.; AIELLO, A.; SRYGLEY, R. B. Early stages of Caligo illioneus and Caligo idomeneus (Nymphalidae, Brassolinae) from Panama, with remarks on larval food plants for the subfamily. Journal of the Lepidopterists' Society, Lawrence, v. 53, p. 142$152,1999$.

PIMENTA, L. H. F. Geologia, Geomorfologia e Pedologia. In: FATMA (Ed.). Parque Estadual da Serra do Tabuleiro: diagnóstico dos meios físico e biótico. Produto básico de zoneamento. Florianópolis: FATMA, 2000. p. 12-43.
REIS, A.; LISBOA, R. Z.; IZA, O. B.; OLIVEIRA, K. N. Flora e vegetação. In: FATMA (Ed.). Parque Estadual da Serra do Tabuleiro: diagnóstico dos meios físico e biótico. Produto básico de zoneamento. Florianópolis: FATMA, 2000. p. 75-118.

RIBEIRO, D. B.; PRADO, P. I.; BROWN JR., K. S.; FREITAS, A. V. L. Temporal diversity patterns and phenology in fruit-feeding butterflies in the Atlantic Forest. Biotropica, v. 42, p. 710-716, 2010.

RITTER, C. D.; LEMES, R.; MORAIS, A. B. B. ; DAMBROS, C. S. Borboletas (Lepidoptera: Hesperioidea e Papilionoidea) de fragmentos de Floresta Ombrófila Mista, Rio Grande do Sul, Brasil. Biota Neotropica, v. 11, p. 361-368, 2011.

RYDON, A. Notes on the use of butterfly traps in East Africa. Journal of the Lepidopterists' Society, Lawrence, v. 18, p. 51-58, 1964.

SALIÉS, E.C. 2000. Apresentação. In: FATMA (Ed.). Parque Estadual da Serra do Tabuleiro: diagnóstico dos meios físico e biótico. Produto básico de zoneamento. Florianópolis: FATMA, 2000. p. 2-10.

SANTOS, E. C.; MIELKE, O. H. H.; CASAGRANDE, M. M. Inventários de borboletas no Brasil: estado da arte e modelo de áreas prioritárias para pesquisa com vistas à conservação. Natureza e Conservação, Curitiba, v. 6, p. 68-90, 2008.

SANTOS, J. P.; ISERHARD, C. A.; TEIXEIRA, M. O.; ROMANOWSKI, H. P. Guia de Borboletas Frugívoras das Florestas Ombrófilas Densa e Mista do Rio Grande do Sul, Brasil. Biota Neotropica, v.11, 2011.

SINGER, M. C. Butterfly-hostplant relationships: host quality, adult choice and larval success. In: VANE-WRIGHT, R. I.; ACKERY, P. E. (Ed.). The biology of butterflies. Florida: Academic Press Inc., 1984. p. 81-88.

UEHARA-PRADO, M.; BROWN JR., K. S.; FREITAS, A. V. L. Biological traits of frugivorous butterflies in a fragmented and a continuous landscape in the south Brazilian Atlantic Forest Journal of the Lepidopterists' Society, Lawrence, v. 59, p. 96 $-106,2005$.

UEHARA-PRADO, M.; FREITAS, A. V. L., FRANCINI, R. B.; BROWN JR., K. S. Guia das borboletas frugívoras da Reserva Estadual do Morro Grande e região de Caucaia do Alto, Cotia (São Paulo). Biota Neotropica, Campinas, v. 4, p. 1-25, 2004.

WAHLBERG, N.; LENEVEU, J.; KODANDARAMAIAH, U.; PEÑA, C.; NYLIN, S.; FREITAS, A. V. L.; BROWER, A. V. Z. Nymphalid butterflies diversify following near demise at Cretaceous/Tertiary boundary. Proceedings of the Royal Society B, London, v. 276, p. 4295-4302, 2009. 